\title{
LETTER OPEN \\ Discovery of a highly potent and selective Bruton's tyrosine kinase inhibitor avoiding impairment of ADCC effects for B-cell non-Hodgkin lymphoma
}

Signal Transduction and Targeted Therapy (2020)5:200

\section{Dear Editor,}

Bruton's tyrosine kinase (BTK) plays a crucial role in the B-cell receptor $(B C R)$ signaling which is essential for B-cell proliferation, differentiation, and cell migration. Aberrant BCR activation has been identified as a major pathogenic factor in several B-cell nonHodgkin lymphoma (B-NHL) subtypes, including diffuse large Bcell lymphoma (DLBCL), mantle cell lymphoma $(M C L)$, follicular lymphoma (FL), and chronic lymphocytic leukemia (CLL). ${ }^{1}$ Therefore, BTK has been recognized as a validated therapeutic target for B-cell malignancies. Ibrutinib, the first approved BTK inhibitor that binds irreversibly to cysteine residue 481, has shown potent clinical activity in the majority of CD20 positive B-cell malignancies. ${ }^{2}$ However, due to the inhibition of off-target kinases such as EGFR, ITK, and TXK, which have a cysteine residue at the identical position of Cys481 of BTK, Ibrutinib also results in some adverse events, such as the antagonizing Rituximab-dependent NK-cell-mediated antibody-dependent cell-mediated cytotoxicity (ADCC) due to its irreversible binding to ITK, which is required for FcR-stimulated NK cell function. ${ }^{3}$ Although several secondary generation inhibitors have shown improved selectivity, ${ }^{4,5}$ more pharmacologically diverse novel inhibitors are still highly demanded in the clinic.

Here, we report the discovery of a novel covalent BTK inhibitor, CHMFL-BTK-85 (abbreviated as compd. 85) (chemical structure shown in Fig. 1a), which achieves high potency against BTK and selectivity over other protein kinases. The ADP-Glo ${ }^{T M}$ biochemical assay with purified BTK protein showed that compd. 85 exhibited an $\mathrm{IC}_{50}$ value of $11.5 \mathrm{nM}$ against BTK which was over 15 -fold more potent than the reversible version compound CHMFL-BTK-85R (chemical structure shown in Supplementary Fig. S1 and Fig. 1b), indicating a covalent binding mode. Immunoblotting analysis of the autophosphorylation at Y223 site of BTK wt and C481S mutant in the transiently transfected HEK293 cells also proved that the covalent binding was via the Cysteine 481 residue (Fig. 1c). To further confirm that compd. 85 could covalently bind to wide-type native BTK in cell, we then conducted a target-engagement assay using the biotinylated analog of compd. 85, i.e., CHMFL-BTK-85B (Supplementary Fig. S2), in REC-1 cell which expresses native BTK kinase. The data showed that after $2 \mathrm{~h}$, compd. 85 could dosedependently compete with CHMFL-BTK-85B and at $1 \mu \mathrm{M}$ concentration it could almost completely covalently label all of the available BTK (Supplementary Fig. S3). The ADP-Glo ${ }^{\text {TM }}$ biochemical assay with kinases targeted by lbrutinib showed that compd. 85 only exhibited moderate selectivity over BMX (10-fold), but it achieved more than 1000-fold selectivity over all of the others including ITK, BLK, EGFR, HER2, HER4, JAK3, and TXK kinases (Fig. 1d and Supplementary Table S1). To further investigate the selectivity of compd. 85 in the kinome, we then tested it

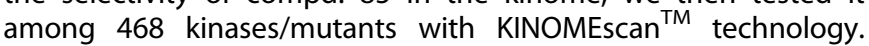

\author{
; https://doi.org/10.1038/s41392-020-00309-1
}

The results showed that it was highly selective $(S$ Score $(1)=0.00)$ at the concentration of $1 \mu \mathrm{M}$ and only BTK kinase was revealed as the strong binding target (Fig. 1e and Supplementary Table S2).

Given the fact that Ibrutinib could impair anti-CD20 antibodies exerted antibody drug-dependent NK-cell-mediated cytotoxicity (ADCC) due to the selectivity problem, we then examined compd. 85 in the human NK cells co-cultured with the Mino cells or SK-OV3 cells in the presence of Rituximab or Herceptin. Ibrutinib strongly inhibited Rituximab and Herceptin-induced IFN- $\gamma$ secretion in the NK cells in a dose-dependent manner between 0.1 and $3 \mu \mathrm{M}$, meanwhile compd. 85 showed no apparent inhibition up to $3 \mu \mathrm{M}$, which recapitulated its weak ITK inhibitory activity (Fig. If and Supplementary Fig. S4). Furthermore, Ibrutinib significantly impaired the antibody-dependent NK-cell-mediated cytotoxicity (ADCC) against Mino and SK-OV-3 cells in the in vitro lactate dehydrogenase (LDH) release experiment (Fig. $1 \mathrm{~g}$ and Supplementary Fig. S5). In comparison, compd. 85 did not affect the killing efficacy of NK cells which further confirmed that it would not abrogate the ADCC effect.

We next evaluated the anti-proliferative effects of compd. 85 against a panel of B-cell lymphoma cell lines. Overall, it was potent to all these cell lines $\left(\mathrm{Gl}_{50} \mathrm{~s}:<2 \mu \mathrm{M}\right)$ while Ibrutinib exhibited a relatively random trend (Fig. $1 \mathrm{~h}$ and Supplementary Table S3). In addition, it displayed similar potency to the second generation BTK kinase inhibitor Acalabrutinib in TMD8 (DLBCL) and REC-1 (MCL) cells. In TMD8, REC-1, and DOHH2 cells, compd. 85 potently blocked the BTK Y223 autophosphorylation $(<10 \mathrm{nM})$ and inhibited the phosphorylation of downstream mediators such as PLCY2, ERK, AKT (Fig. 1i), and p-NF-KB p65 (Supplementary Fig. S6). In addition, dose-dependent apoptotic induction and cell cycle arrest were observed in these cell lines (Supplementary Fig. S7a, b).

The in vivo pharmacokinetic study showed that compd. 85 bore acceptable bioavailability $(F=29 \%)$ and suitable half-life $\left(T_{1 / 2}=\right.$ $2.9 \mathrm{~h}$ ), and good drug exposure $\left(\mathrm{AUC}_{0_{-\mathrm{t}}}=2145 \mathrm{ng} / \mathrm{mL}\right.$ ) for oral administration at $10 \mathrm{mg} / \mathrm{kg}$ in rats (Table S4). The dose escalation study showed that compd. 85 was well tolerated up to $800 \mathrm{mg} / \mathrm{kg} /$ day dosage for continuous 14 days with no apparent toxicity observed (Supplementary Fig. S8a, b). In addition, compd. 85 exhibited dose-dependent anti-tumor efficacy in the TMD8 cell (DLBCL)-inoculated xenograft mouse model and the tumor growth inhibition (TGI) of $96 \%$ was achieved at $100 \mathrm{mg} / \mathrm{kg} /$ day dosage, which was better than lbrutinib $(\mathrm{TGI}=90 \%)$ at the same dosage (Fig. 1j). Again, no weight loss or any other obvious signs of toxicity were observed (Supplementary Fig. S9a). In the TMD8 tumor tissues, the BTK-mediated signaling was dose-dependently inhibited by compd. 85, which was consistent with its in vivo antitumor phenotype and confirmed its on-target effect 


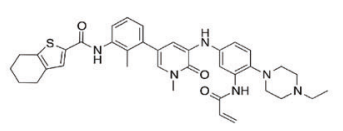

CHMFL-BTK-85 b

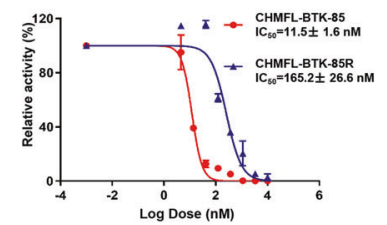

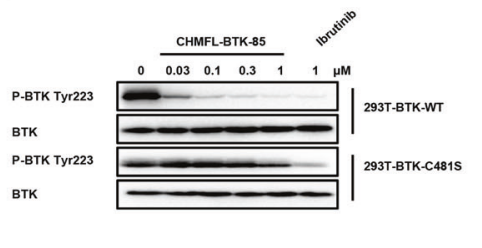

d

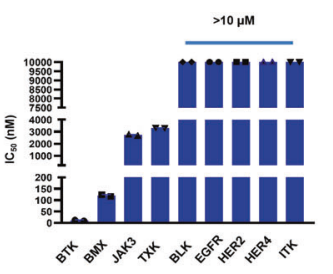

e

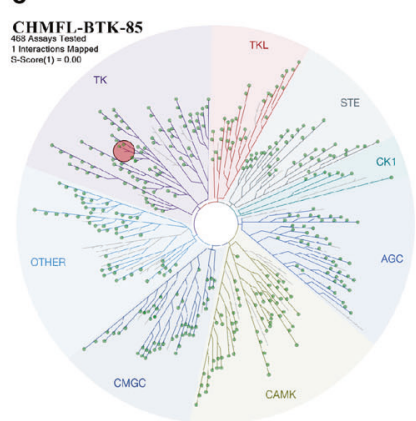

h

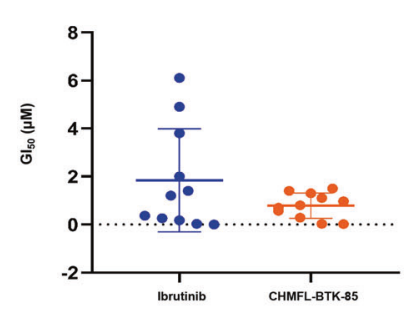

j

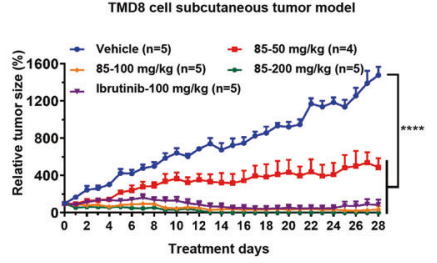

f

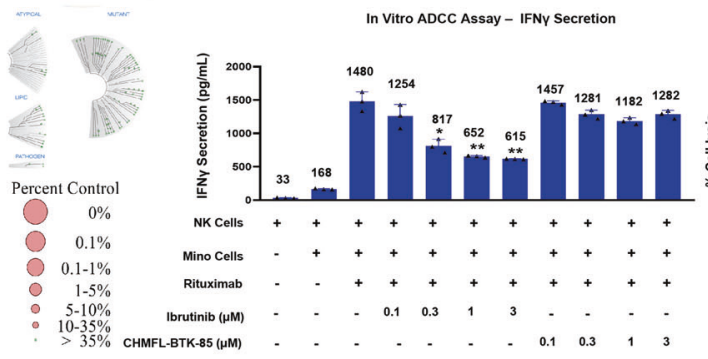

g

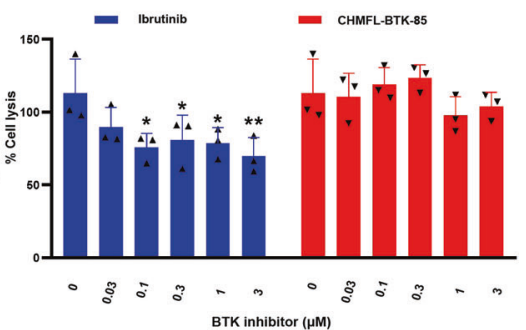

i
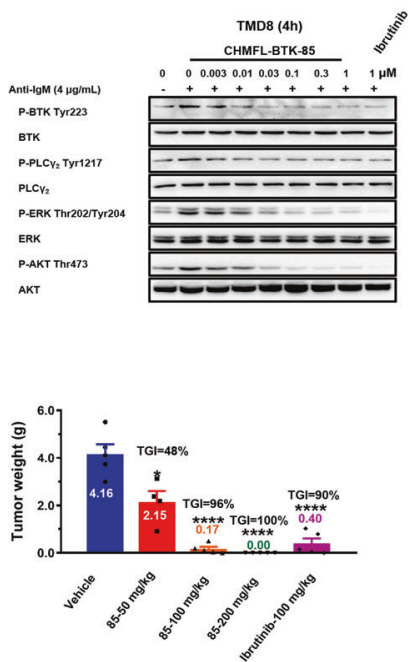
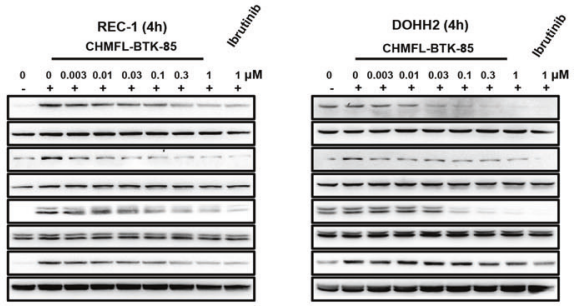

k

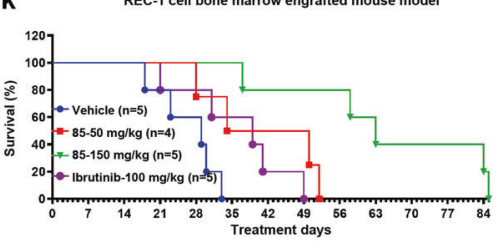

Fig. 1 CHMFL-BTK-85 could avoid impairment of the ADCC effects in B-cell non-Hodgkin lymphoma. a Chemical structure of CHMFL-BTK-85. $\mathbf{b}$ ADP-Glo ${ }^{\text {TM }}$ assay determination of the $\mathrm{IC}_{50}$ values of CHMFL-BTK-85 and CHMFL-BTK-85R against BTK kinase. c CHMFL-BTK-85 inhibited the cellular autophosphorylation Tyr223 of WT BTK and the C481S mutant in transiently transfected HEK293 cells. The blots were cropped for improved clarity and conciseness. d ADP-Glo ${ }^{\text {TM }}$ assay determination of the IC 50 values of CHMFL-BTK-85 against BTK, BLK, BMX, EGFR, HER2, HER4, ITK, JAK3, and TXK kinases. e Kinome-wide selectivity profiling of CHMFL-BTK-85 with DiscoverX's KINOMEscan ${ }^{\text {TM }}$ technology (http:// $^{-1}$ www.kinomescan.com). The red circles indicated kinases bound, and circle size indicated relative binding affinity compared to DMSO (Ctrl\%). The complete dataset was shown in Supplementary Table 2. f Mino cells and NK cells were co-seeded and treated with vehicle or various concentrations of BTK inhibitors in the presence of Rituximab; interferon (IFN)- $\gamma$ levels in the conditioned medium were measured as a readout of the assay. $\mathbf{g}$ Mino cells and NK cells were co-seeded and treated with vehicle or various concentrations of Ibrutinib and CHMFL-BTK85 in the presence of Rituximab. Cytotoxicity of the target cells was determined by lactate dehydrogenase release into the culture medium. h Anti-proliferative effects of CHMFL-BTK-85 against a panel of B-cell lymphoma cell lines. The cells were treated with Ibrutinib and CHMFLBTK-85 (maximum concentration $10 \mu \mathrm{M}$ ) for $72 \mathrm{~h}$, and then cell viability was measured using the CellTiter-Glo assay (Error bars, mean \pm SEM, $n$ $=3$ ). $\mathrm{i}$ The phosphorylation levels of BTK (Tyr223), PLC $\gamma_{2}$ (Tyr1217), ERK1/2 (Thr202/Tyr204), and AKT (Ser473) were detected by western blot in TMD8, REC-1, and DOHH2 cell lines. These cells were incubated with the indicated concentrations of CHMFL-BTK-85 for $4 \mathrm{~h}$ before lysis. $\mathbf{j}$ Antitumor effects of CHMFL-BTK-85 with once daily (QD) dosing at 50, 100, and $200 \mathrm{mg} / \mathrm{kg}$. The total study length was 28 days. (Left) Representative graphs of relative tumor size are shown. (Right) Representative graphs of tumor weight of different groups are shown. $\mathbf{k}$ Antitumor efficacy of CHMFL-BTK-85 in the bone marrow engrafted mouse model. Kaplan-Meier plots of survival. The disseminated NOD/SCID mice were intravenously inoculated with REC-1 cells and received daily oral administration of CHMFL-BTK-85 dosing at 50 and $150 \mathrm{mg} / \mathrm{kg}$. The total study length was 84 days, and each treatment group contained $4-5$ animals. Date are shown as mean $\pm \mathrm{SEM}$, ${ }^{*} P$-value $<0.05$, ${ }^{* *} P$-value $<$ $0.01,{ }^{* * *} P$-value $<0.001$, and ${ }^{* * * *} P<0.0001$

(Supplementary Fig. S9b). In order to further evaluate the in vivo efficacy of compd. 85, we then examined it in the REC-1 cell (MCL)inoculated xenograft mouse model, $100 \mathrm{mg} / \mathrm{kg} /$ day dosage of compd. 85 slowed down the tumor progression and showed a TGI of $65 \%$ without obvious signs of toxicity, which was slightly better than Ibrutinib $(\mathrm{TGI}=59 \%)$ and Acalabrutinib $(\mathrm{TGI}=58 \%)$ at the same dosage (Supplementary Fig. S10a, b). In total, $150 \mathrm{mg} / \mathrm{kg} / \mathrm{day}$ dosage of compd. 85 could achieve TGl of $79 \%$. Evaluation of drug 
enrichment in the tumor showed that at the same dosage $(100 \mathrm{mg} / \mathrm{kg})$ compd. 85 could reach a much higher concentration $(2.37 \mu \mathrm{M})$ relative to Ibrutinib $(1.23 \mu \mathrm{M})$ and Acalabrutinib $(1.30 \mu \mathrm{M})$. This better in tumor PK profile may partially explain the better in vivo efficacy of compd. 85 . In the REC-1 cell-mediated orthogonal mouse model of bone marrow engraftment, compd. 85 dose-dependently extended the median survival time of mice to 42 days at $50 \mathrm{mg} / \mathrm{kg} /$ day dosage and meanwhile exhibited better efficacy than Ibrutinib (median survival time was 39 days at $100 \mathrm{mg} / \mathrm{kg} /$ day dosage). At $150 \mathrm{mg} / \mathrm{kg} /$ day dosage, compd. 85 could even extend the median survival time of mice to 63 days (Fig. 1k and Supplementary Fig. S10c).

In short, we have discovered a novel highly selective covalent BTK kinase inhibitor CHMFL-BTK-85, which did not affect the NKcell-mediated ADCC effects and showed good in vitro and in vivo anti-tumor efficacies. These data support further investigation of CHMFL-BTK-85 as a potential clinical drug candidate, especially in combination with anti-CD20 antibodies, for B-cell non-Hodgkin lymphoma.

\section{DATA AVAILABILITY}

The datasets used and/or analyzed to support the findings of this study are available in this paper or the Supplementary Information. Any other raw data that support the findings of this study are available from the corresponding author upon reasonable request.

\section{ACKNOWLEDGEMENTS}

This work was supported by the National Natural Science Foundation of China (Grant Nos. 81773777, 81872748, and 81803366), the National Science \& Technology Major Project "Key New Drug Creation and Manufacturing Program" of China (Grant No. 2018ZX09711002), the Natural Science Foundation of Anhui Province (Grant No. 1808085MH268), the China Postdoctoral Science Foundation (Grants Nos. 2018T110634 and 2018M630720), the Frontier Science Key Research Program of CAS (Grant No. QYZDB-SSW-SLH037), the Innovative Program of Development Foundation of Hefei Center for Physical Science and Technology (Grant No. 2019HSCCIP011), the CASHIPS Director's Fund (Grant No. BJPY2019A03), and the Key Program of 13th five-year plan of CASHIPS (Grant No. KP-2017-26). We are also grateful for the support of Hefei leading talent for F.Z.

\section{ADDITIONAL INFORMATION}

The online version of this article (https://doi.org/10.1038/s41392-020-00309-1) contains supplementary material, which is available to authorized users.

Competing interests: The authors declare no competing interests.
Juan Liu ${ }^{1,2}$, Qianmao Liang ${ }^{1,2}$, Aoli Wang ${ }^{1,3}$, Fengming Zou ${ }^{1,3}$, Ziping $\mathrm{Qi}^{1,3}{ }^{1}$, Kailin $\mathrm{Yu}^{1}$, Qingwang Liu ${ }^{1,3,4,5}$, Cheng Chen ${ }^{1,2,3^{\prime}}$ Jing Liu ${ }^{1,2,3}$ and Qingsong Liu (iD ${ }^{1,2,3,4,5,6}$

${ }^{1}$ Anhui Province Key Laboratory of Medical Physics and Technology, Institute of Health and Medical Technology, Hefei Institutes of Physical Science, Chinese Academy of Sciences, 230031 Hefei, Anhui, P. R. China; ${ }^{2}$ University of Science and Technology of China, 230036 Hefei, Anhui, P. R. China; ${ }^{3}$ Hefei Cancer Hospital, Chinese Academy of Sciences, 230031 Hefei, Anhui, P. R. China; ${ }^{4}$ Precision Medicine Research Laboratory of Anhui Province, 230088 Hefei, Anhui, P. R.

China; ${ }^{5}$ Precision Targeted Therapy Discovery Center, Institute of Technology Innovation, Hefei Institutes of Physical Science, Chinese Academy of Sciences, 230088 Hefei, Anhui, P. R. China and ${ }^{6}$ Institutes of Physical Science and Information Technology, Anhui University, 230601 Hefei, Anhui, P. R. China

These authors contributed equally: Juan Liu, Qianmao Liang, Aoli Wang, Fengming Zou.

Correspondence: Jing Liu (jingliu@hmfl.ac.cn) or Qingsong Liu (qsliu97@hmfl.ac.cn)

\section{REFERENCES}

1. Pal Singh, S., Dammeijer, F. \& Hendriks, R. W. Role of Bruton's tyrosine kinase in B cells and malignancies. Mol. Cancer. https://doi.org/10.1186/s12943-018-0779-z (2018).

2. Mathur, R. Burton's Tyrosine Kinase Inhibition by Ibrutinib: current status. J. Leuk. https://doi.org/10.4172/2329-6917.1000e113 (2015).

3. Kohrt, H. E. et al. Ibrutinib antagonizes rituximab-dependent NK cell-mediated cytotoxicity. Blood 123, 1957-1960 (2014).

4. Byrd, J. C. et al. Acalabrutinib (ACP-196) in relapsed chronic lymphocytic leukemia. New Engl. J. Med. 374, 323-332 (2016).

5. Guo, Y. et al. Discovery of zanubrutinib (BGB-3111), a novel, potent, and selective covalent inhibitor of Bruton's tyrosine kinase. J. Med. Chem. 62, 7923-7940 (2019).

\begin{abstract}
(c) (i)
Open Access This article is licensed under a Creative Commons Attribution 4.0 International License, which permits use, sharing, adaptation, distribution and reproduction in any medium or format, as long as you give appropriate credit to the original author(s) and the source, provide a link to the Creative Commons license, and indicate if changes were made. The images or other third party material in this article are included in the article's Creative Commons license, unless indicated otherwise in a credit line to the material. If material is not included in the article's Creative Commons license and your intended use is not permitted by statutory regulation or exceeds the permitted use, you will need to obtain permission directly from the copyright holder. To view a copy of this license, visit http://creativecommons. org/licenses/by/4.0/.
\end{abstract}

(c) The Author(s) 2020 